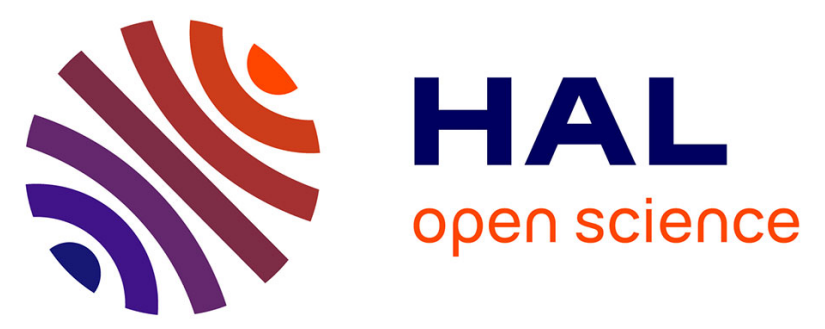

\title{
Hot-carrier evaluation of a zero-cost transistor developed via process optimization in an embedded non-volatile memory CMOS technology
}

P. Devoge, Hassen Aziza, P. Lorenzini, F. Julien, A. Marzaki, A. Malherbe, M. Mantelli, T. Cabout, J. Delalleau, S. Haendler, et al.

\section{To cite this version:}

P. Devoge, Hassen Aziza, P. Lorenzini, F. Julien, A. Marzaki, et al.. Hot-carrier evaluation of a zero-cost transistor developed via process optimization in an embedded non-volatile memory CMOS technology. Microelectronics Reliability, 2021, 126, pp.114265. 10.1016/j.microrel.2021.114265 . hal03500203

\section{HAL Id: hal-03500203 \\ https://hal.science/hal-03500203}

Submitted on 6 Jan 2022

HAL is a multi-disciplinary open access archive for the deposit and dissemination of scientific research documents, whether they are published or not. The documents may come from teaching and research institutions in France or abroad, or from public or private research centers.
L'archive ouverte pluridisciplinaire HAL, est destinée au dépôt et à la diffusion de documents scientifiques de niveau recherche, publiés ou non, émanant des établissements d'enseignement et de recherche français ou étrangers, des laboratoires publics ou privés. 


\title{
Hot-carrier evaluation of a zero-cost transistor developed via process optimization in an embedded non-volatile memory CMOS technology
}

\author{
P. Devogea ${ }^{a, b, c^{*}}$, H. Aziza ${ }^{b}$ P. Lorenzinic, F. Julien ${ }^{a}$, \\ A. Marzaki ${ }^{\mathrm{a}}$, A. Malherbe ${ }^{\mathrm{a}}$, M. Mantellia ${ }^{\mathrm{a}}$ T. Cabout ${ }^{\mathrm{d}}$, \\ J. Delalleau ${ }^{\text {a }}$ S. Haendler ${ }^{\mathrm{d}}$, A. Regnier ${ }^{\mathrm{a}}$, S. Niel ${ }^{\mathrm{d}}$ \\ ${ }^{a}$ STMicroelectronics, Rousset, France \\ ${ }^{\mathrm{b}}$ Aix-Marseille University, CNRS, IM2NP UMR 7334, Marseille, France \\ ' University of Côte d'Azur, Polytech'Lab UPR UCA 7498, Sophia-Antipolis, France \\ d STMicroelectronics, Crolles, France
}

\begin{abstract}
A new transistor architecture is developed by reusing already existing fabrication process bricks in an embedded non-volatile memory (eNVM) sub-40 nm CMOS technology, resulting in a middle-voltage zero-cost transistor, ideal for low-cost products. TCAD simulations are undertaken to confirm the feasibility of the process optimization and predict the transistor performance and reliability. The new transistor is fabricated then electrically characterized. The new device shows good analogue performances for no cost added. A hot-carrier injection (HCI) degradation evaluation is performed and confirms the reliability of the device.
\end{abstract}

\section{Keywords}

Transistor, MOSFET, CMOS, zero-cost, analogue, middle-voltage, hot-carrier, HCI, reliability

\section{Introduction}

In low-cost and high-volume products, such as smartcards and Internet of Things (IoT) devices, lowcost transistor architectures are highly desirable [1]. An ideal CMOS technology contains a multitude of transistor architectures, each one addressing different voltage ranges, with adjusted implants, oxide thicknesses, and spacers (Fig. 1a). A small transistor is used for digital areas, a larger one for input/output (IO) functions. With embedded Non-Volatile Memory (eNVM), it is even more complex, as specific transistors are needed to handle memory-related High-Voltage (HV) operations [2]. This leads to a high number of photomasks, and dedicated process steps for each device, increasing the cost of the technology.

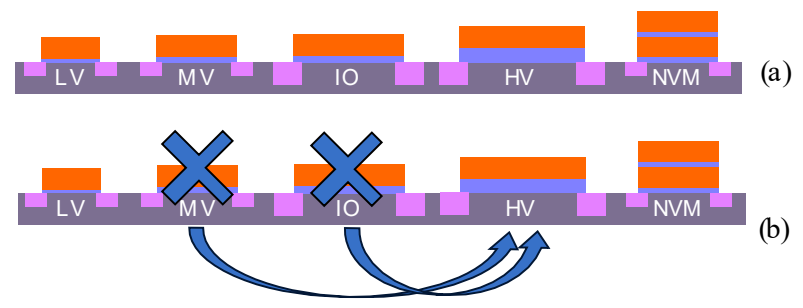

Fig. 1 - (a) Device architectures used in a standard eNVM technology. (b) Reduction of the number of Device architectures in a low-cost eNVM technology.
To minimize the cost of a technology, the number of process steps and photomasks must be reduced. Consequently, only a small subset of the different transistor architectures presented in Fig. 1a are used. In the targeted sub-40 nm eNVM low-cost technology, a single $\mathrm{HV}$ transistor architecture is used as an alternative to the IO and Middle-Voltage (MV) transistors for all circuit designs that need to withstand a voltage higher than the one supported by the Low-Voltage (LV) digital transistor, as shown in Fig. 1b. This approach is not optimized, as $\mathrm{HV}$ transistors are designed firstly to withstand high-voltages, and not necessarily to have good analogue performances, or to provide the smallest silicon footprint. Thus, there is a need for a "zero-cost" device to address the MV range (between 3 and $5 \mathrm{~V}$ ), which is often neglected in favour of $\mathrm{LV}$ or $\mathrm{HV}$ transistors in low-cost technology.

In this work, we investigate the possibility of reusing already existing process bricks (oxide, implants, spacers), to build a new zero-cost transistor for the MV range that have better analogue performances compared to the HV transistor. In section 2 the proposed zero-cost process optimization is presented. TCAD simulation of the new transistor is undertaken, including transistor performance and reliability prediction. The results are presented in section 3 . In section 4 the newly fabricated MV transistor is electrically characterised and compared to the existing $\mathrm{HV}$ transistor. Finally, hot-carrier injection (HCI) stress tests of the transistors are performed and presented in section 5 .

* Corresponding author. paul.devoge@st.com 


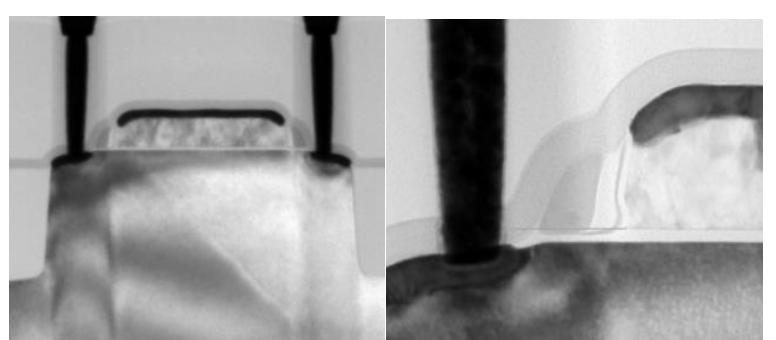

Fig. 2 - Transmission Electron Microscopy (TEM) images of the new MV device

\section{Presentation of the new transistor architecture}

The new MV transistor reuses most of the process steps of the available HV device, which is engineered to withstand voltages up to $10 \mathrm{~V}$ on its source, drain and gate. For that, a thick gate oxide, large spacers, anti-punch-through well implants and large lightlydoped-drain (LDD) implants are used. These process features are relevant when dealing with high-voltage, but not necessarily optimized for low or middle-voltage. Compared to the classical HV transistor, our new MV device uses a thinner oxide, that already serves as a tunnel oxide in the eNVM available in the technology. Fig. 2 shows the Transmission Electron Microscopy (TEM) images of our new MV device.

The gate oxide thickness is reduced by $40 \%$, which has the immediate effect of increasing the gate capacitance $\mathrm{C}_{\mathrm{OX}}$, thus lowering the threshold voltage $\mathrm{V}_{\mathrm{T}}$, and increasing the saturated drive current ION. Those effects are highly desirable for analogue applications. On the other hand, the gate oxide thickness reduction is usually accompanied by a higher OFF-state leakage current IOFF. An enhancement of the transistor matching parameter $A_{V T}$ is also predicted, since it is known to be proportional to the gate oxide thickness $[3,4]$.

\section{TCAD simulation}

Technology Computer Aided Design (TCAD) simulations are performed to verify the feasibility of the fabrication process, to predict the electrical characteristics of the new transistor, and the expected performance gains compared to the HV transistor. Twodimensional static simulations are made using the Synopsys Sentaurus TCAD suite [5, 6]. During electrical behaviour simulation, the electrostatic potential is modelled using the Fermi-Dirac statistic. The driftdiffusion model is used to model the carrier transport. Mobility is modelled using the Philips unified mobility model (PhuMob) [7]. Shockley-Read-Hall (SRH) recombination is taken into account, including doping and electric field correction. Finally, avalanche generation (impact ionisation) is modelled using the new University of Bologna impact ionization model (UniBo2) [8].

\subsection{Device performance metrics}

Important device parameters are the linear and saturated threshold voltages $\mathrm{V}_{\text {T-LIN }}$ and $\mathrm{V}_{\mathrm{T} \text {-SAT }}$, measured at a fixed constant drain current $I_{D}$ equal to $100 \mathrm{nA}$ multiplied by the polysilicon gate width/length ratio $\mathrm{W} / \mathrm{L}$, and with a drain-source voltage $\mathrm{V}_{\mathrm{DS}}$ equal to $0.1 \mathrm{~V}$ and $3.3 \mathrm{~V}$, respectively. We are particularly interested in the variations of these parameters with the gate length $\mathrm{L}$ of the device, as it can highlight Short Channel Effects (SCE) [9]. We will also study the ON-state saturated drain current $\mathrm{I}_{\mathrm{ON}}$ at a $\mathrm{V}_{\mathrm{DS}}$ and gate-source voltage $\mathrm{V}_{\mathrm{GS}}$ both of $3.3 \mathrm{~V}$, and the OFF-state leakage current $\mathrm{I}_{\mathrm{OFF}}$ at a $V_{D S}$ of $3.3 \mathrm{~V}$. Finally, we will examine the drain-source breakdown voltage $B_{V D S S}$, defined as the $V_{D S}$ value for which $\mathrm{I}_{\mathrm{D}}$ is equal to $1 \mathrm{nA}$, with the source, gate and bulk grounded.

\subsection{Fabrication process TCAD simulation}

The first stage of the TCAD simulation is the fabrication process simulation. The fabrication steps such as photolithography, etching, deposition, implantation, oxidation are simulated using physical equations modelling these phenomena, along with the layout sketch of the device's structure. The device is discretized (meshed) into a finite element structure. Fig. 3a shows TCAD simulation results for the new MV transistor, with a gradient colourmap of the doping concentration. The comparison of the TCAD snapshots with the TEM pictures of Fig. 2 confirms the good agreement between the TCAD simulation and the actual fabrication process.

\subsection{Electrical behaviour simulation}

The second stage of TCAD simulations is the electrical behaviour simulation of the transistor. This operation takes as input the meshed device generated by the fabrication process simulation. The simulator solves at each mesh point the fundamental physical equations describing the device operation, such as the electrical transport equations. By applying the same voltages used during electrical device characterisation and presented in section 3.1, TCAD simulation can predict the performance of our new MV transistor compared to the HV device.

The threshold voltages $\mathrm{V}_{\text {T-LIN }}$ and $\mathrm{V}_{\mathrm{T}-\mathrm{SAT}}$ of the $\mathrm{HV}$ and the new MV device have been simulated for different gate lengths (L) ranging from 0.5 to $10 \mu \mathrm{m}$, with a width (W) of $10 \mu \mathrm{m}$. The results for NMOS are shown in Fig. $3 b$. These results confirm the expected $V_{T}$ drop associated with thinner gate oxide, close to a rigid curve shift. The $\mathrm{V}_{\mathrm{T}}$ curve of our new MV device is in an interesting range above $200 \mathrm{mV}$. This value is low (good for analogue applications) but high enough to account for the decrease in $\mathrm{V}_{\mathrm{T}}$ with temperature, as the transistor should still have a positive $\mathrm{V}_{\mathrm{T}}$ at $125^{\circ} \mathrm{C}$. For $\mathrm{V}_{\mathrm{T}-\mathrm{SAT}}$ (dark blue in Fig. 3b, TCAD and measurements are 
(a)
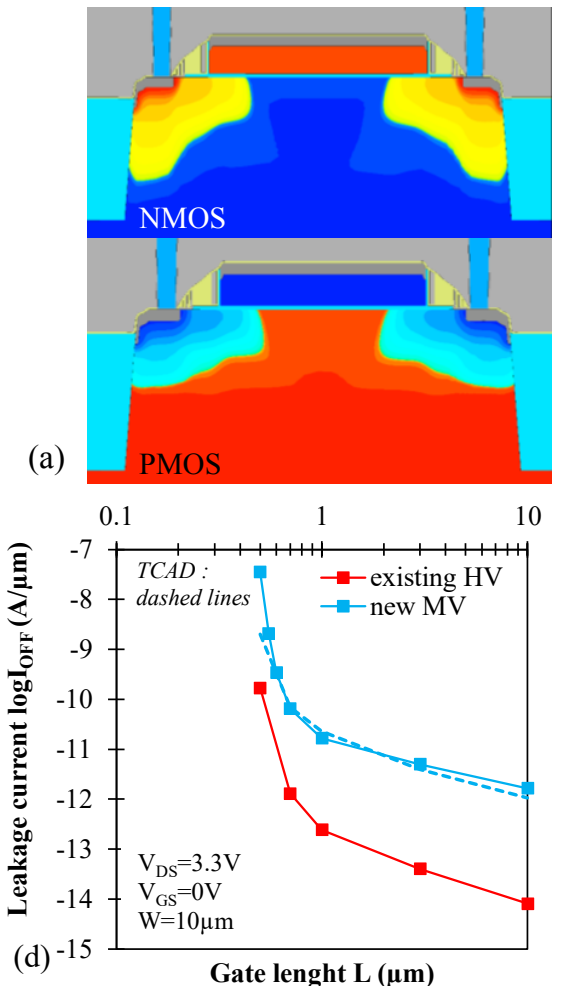

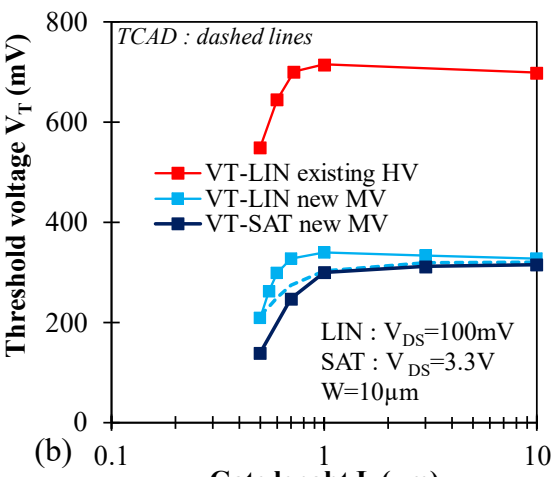

Gate lenght $\mathrm{L}(\boldsymbol{\mu m})$

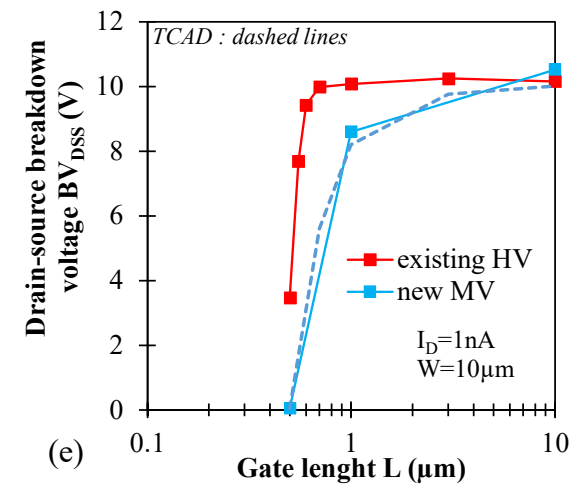

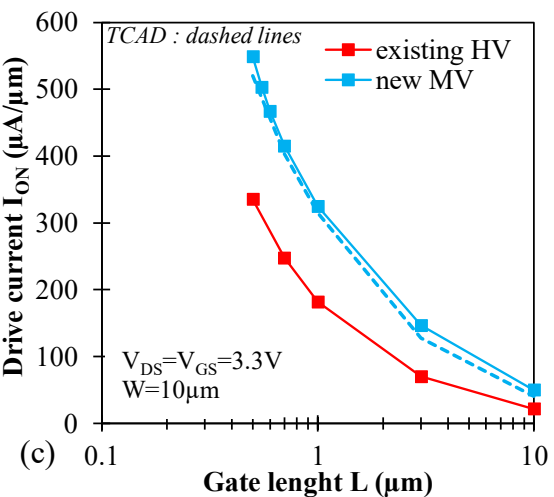

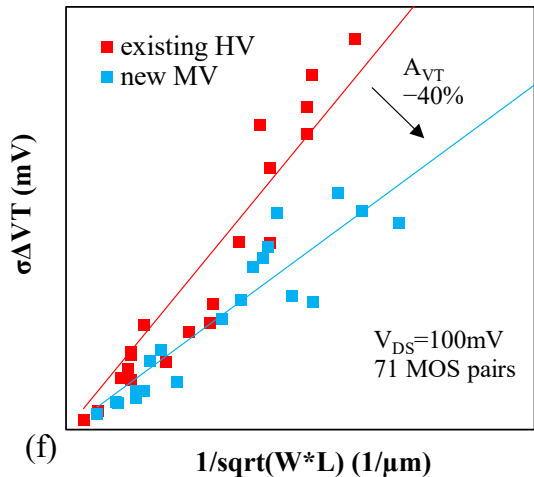

Fig. 3 - TCAD and silicon results. From top to bottom and left to right: (a) TCAD snapshots with doping gradient colourmap (NMOS on the top and PMOS on the bottom), (b) $\mathrm{V}_{\mathrm{T}}$ vs L (dashed lines are from TCAD simulations, solid lines are silicon measurements), (c) Ion vs L, (d) Ioff vs L, (e) BVDss vs L, and (f) Pelgrom plot (transistor matching),

superimposed), the new MV transistor undergo DrainInduced Barrier Lowering (DIBL) [10], which reduces the $\mathrm{V}_{\mathrm{T}}$ when the drain voltage is high. DIBL and SCE limit the minimal gate length $\mathrm{L}_{\min }$ to around $0.7 \mu \mathrm{m}$.

The saturated $\mathrm{ON}$-state drive current $\mathrm{I}_{\mathrm{ON}}$ has been simulated with the same TCAD setup. NMOS results are shown in Fig. 3c. The expected gain in the drive current associated with thinner gate oxide is confirmed. For the same polarization, a device with a lower $\mathrm{V}_{\mathrm{T}}$ has a higher drain current. This is a second advantage of the proposed device.

The OFF-state leakage current $\mathrm{I}_{\mathrm{OFF}}$ has been simulated (Fig. 3d), and as expected, it is higher because of the thinner gate oxide and lower $\mathrm{V}_{\mathrm{T}}$. This parameter also limits the $\mathrm{L}_{\min }$ to around $0.7 \mu \mathrm{m}$ to stay below our maximum acceptable leakage current value of $100 \mathrm{pA} / \mu \mathrm{m}$.

The drain-source breakdown voltage $\mathrm{BV}_{\mathrm{DSS}}$ has been simulated (Fig. 3e). BV $V_{\text {DSS }}$ has several contributors including drain-bulk reverse diode leakage current and OFF-state channel leakage current [11]. For large L, the main contributor is the drain-bulk junction leakage current, so a thinner gate oxide has minor impact on $\mathrm{BV}_{\text {DSS. }}$. When reducing $\mathrm{L}$, the OFF-state channel leakage current increases, lowering the value of the extracted $\mathrm{BV}_{\mathrm{DSS}}$. This is also a consequence of SCE.

TCAD simulation results confirms the functionality of our new MV transistor. This motivates its development as it can be obtained for free in terms of process steps and photomasks. It provides a lower $\mathrm{V}_{\mathrm{T}}$ (an improvement for analogue design), a higher $\mathrm{I}_{\mathrm{ON}}$ compared to the $\mathrm{HV}$ transistor, and it can withstand high drain voltages. The main drawback is that SCE and DIBL limits the $\mathrm{L}$ reduction to no lower than $0.7 \mu \mathrm{m}$ due to $\mathrm{V}_{\mathrm{T}}$ roll-off (which can lead to a negative $\mathrm{V}_{\mathrm{T}}$ at high temperature or high drain voltage) and increased leakage current IOFF.

\subsection{Device reliability prediction}

To predict the hot-carrier injection (HCI) reliability of our new MV transistor compared to the existing HV, several TCAD simulation output data can be used, without the need of a full degradation model calibration. These output data includes the avalanche generation rate (impact ionization), which is dependent of the electric field and the possibility of acceleration, in other words, a sufficiently large space charge region compared to the mean free path of the charge carrier.

Fig 4a shows the electric field intensity (top) and impact ionization (bottom) colourmaps to illustrate in which regions these phenomena are the most intense. The black isolines represent the doping, showing the source and drain junction position. Fig. $4 \mathrm{~b}$ shows the lateral electric field along the channel when the transistor is biased in the saturation region with $\mathrm{V}_{\mathrm{GS}}=\mathrm{V}_{\mathrm{DS}}=3.3 \mathrm{~V}$. 

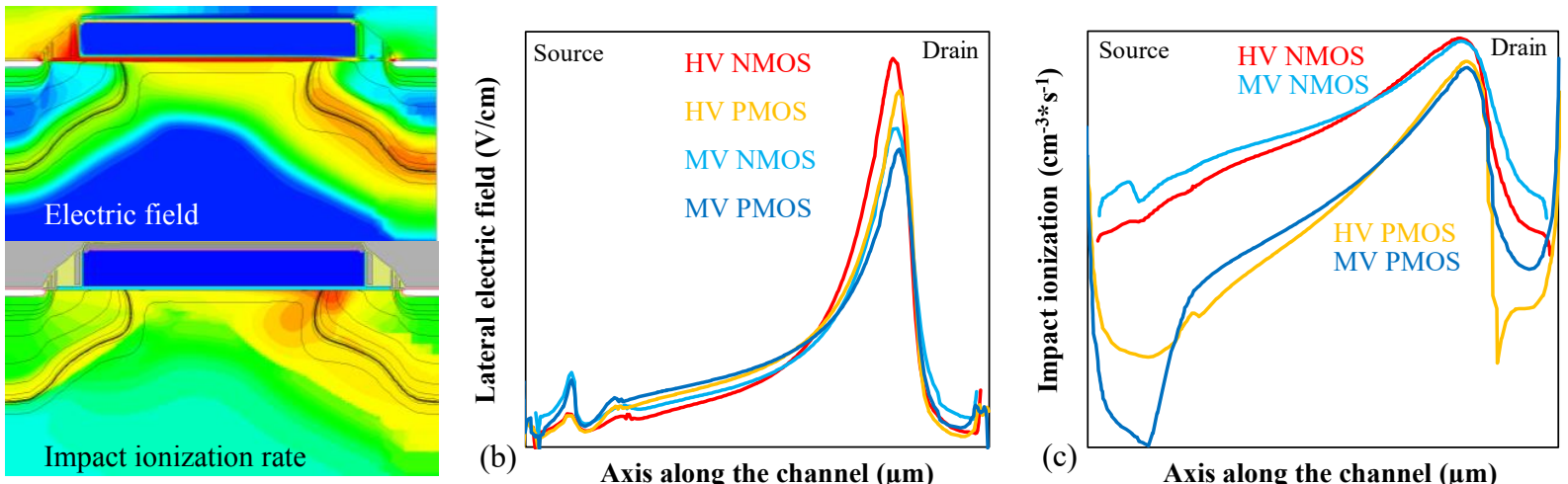

(c)

Axis along the channel $(\mu \mathrm{m})$

Fig. 4 - TCAD data for hot-carrier degradation prediction. (a) TCAD colourmap of electric field (top) and impact ionization rate (bottom). Black isolines are doping concentration to highlight the drain (right side) and source (left side) junction positions. (b) Lateral electric field in the channel. (c) Impact ionization rate in the channel.

The maximum lateral electric is located near the drain region (right side in Fig. 4), and at that maximum, the lateral electric field in the channel of MV transistors is lower than the one in HV transistors (comparing NMOS transistors together and PMOS together). Although the difference is small, if the injection and degradation mechanisms were the same, we could expect a slight increase in the device lifetime for the new MV NMOS transistor. Fig. 4c shows the impact ionisation rate along the channel with the same voltage biasing. Note that this biasing condition may not be the worst case condition for HCI degradation, but it allows us to locate the region where carrier injection would most likely take place. TCAD simulation of Fig. 4c shows a very small difference of electron pair generation between MV and $\mathrm{HV}$ transistors for the considered bias conditions. In this graph, the difference between the NMOS and PMOS in terms of impact ionisation rate can be observed, this is due to the shorter mean free path and higher oxide barrier for holes in PMOS transistors [12].

\section{Experimental results for device parameters}

After promising TCAD results, the new MV device has been fabricated on sub- $40 \mathrm{~nm}$ eNVM technology engineering wafers. Experimental results will be presented in this section.

Experimental results show an overall good agreement with the TCAD simulation results. Fig. 3 presents a comparison between the TCAD and experimental results for the NMOS version of our new MV transistor. PMOS results are not shown here for conciseness but the conclusions are similar.

As predicted by the TCAD simulation, the $\mathrm{V}_{\mathrm{T}}$ of our new MV transistor is lower due to the thinner gate oxide, but compared to the results of the TCAD simulation, the transistor shows a small increase in $\mathrm{V}_{\mathrm{T}}$ with decreasing $\mathrm{L}$ before the roll-off at small $\mathrm{L}$. This phenomenon is known as reverse short channel effect (R-SCE) and can have multiple origins [13]. On the other hand, ION, I OFF and $B_{\text {VDSs }}$ of the fabricated transistor show very good agreement with the TCAD simulation.

Concerning the transistor matching, the threshold voltage matching parameter $A_{v T}$ has been calculated by firstly measuring the standard deviation of the difference of threshold voltages $\sigma_{\Delta \mathrm{VT}}$ of many pairs of identical adjacent transistors. The results are presented in the form of a Pelgrom Plot in Fig. 3f. The $A_{V T}$ dependence with gate oxide thickness is observed, as our new MV device shows an improved AvT compared to the HV device. A small $A_{V T}$ is highly desirable for analogue designs, usually containing a lot of differential pairs and current mirrors, which are fundamental circuit blocks relying on the matching of transistor parameters for optimal performance.

The electrical results obtained using TCAD simulations are confirmed by silicon characterization. By reusing a thinner oxide already available in the technology, we have obtained a new transistor with enhanced performances that can be made for free. It has a lower $\mathrm{V}_{\mathrm{T}}$, a higher $\mathrm{I}_{\mathrm{ON}}$ and a better $\mathrm{A}_{\mathrm{VT}}$ compared to the HV transistor.

\section{Hot-carrier injection (HCI) degradation}

The degradation of the device parameters over time caused by hot-carrier injection (HCI) is important for assessing the reliability of the transistor. During HCI stress, carriers of high energy are generated near the drain where a high electric field exists, and they are injected in the gate oxide where they can be trapped or break Si-H bonds. This phenomenon shifts the transistor parameters such as $\mathrm{V}_{\mathrm{T}}$ and $\mathrm{I}_{\mathrm{ON}}$ by affecting the channel carrier mobility and the effective channel potential $[14$, $15,16]$.

\subsection{HCI degradation measurement setup}

For the evaluation of HCI reliability of new devices, the worst-case bias conditions need to be evaluated during preliminary measurements. The $\mathrm{V}_{\mathrm{DS}}$ at which the HCI stress is applied is usually either the maximum voltage at which the transistor will be used during normal usage plus a small margin to account for possible power supply overshoots, or the voltage at which the breakdown region starts. For a given $\mathrm{V}_{\mathrm{DS}}$, the 

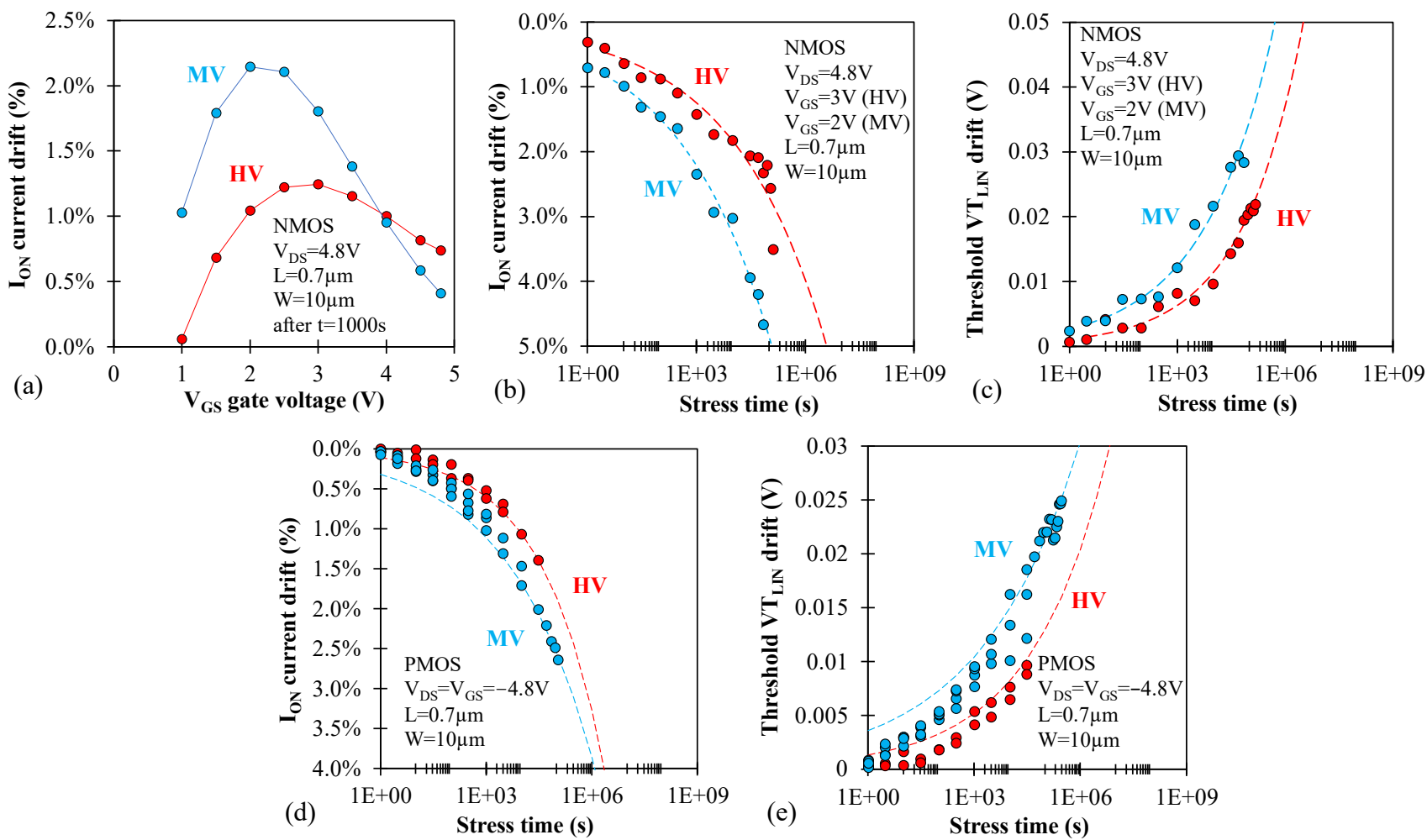

Fig. 5 - HCI experimental results. (a) Ion degradation after $1000 \mathrm{~s}$ of preliminary HCI stress of the NMOS transistors to select the most stressing bias condition. (a) Absolute value of drive current drift over HCI stress time for MV and HV NMOS. (b) Threshold voltage shift over HCI stress time for NMOS. (c) Absolute value of drive current drift over HCI stress time for MV and HV PMOS (d) Absolute value of threshold voltage shift over HCI stress time for PMOS

corresponding $\mathrm{V}_{\mathrm{GS}}$ is chosen to generate the maximum degradation. The worst-case HCI degradation can usually be observed for two different gate voltage conditions:

- maximum gate voltage $\mathrm{V}_{\mathrm{GS}}$

- maximum bulk current $\mathrm{I}_{\mathrm{B}}$

$\mathrm{I}_{\mathrm{B}}$ being proportional to the number of electron-hole pairs produced by hot carrier [14]. In the first case (maximum $\mathrm{V}_{\mathrm{GS}}$, i.e. $\mathrm{V}_{\mathrm{GS}}=\mathrm{V}_{\mathrm{DS}}$ ), hot electrons are injected in the gate (for NMOS transistors, and hot holes for PMOS). This is usually the worst case for PMOS transistors. In the second case, both hot holes and hot electrons are believed to be injected in the gate [12]. This is generally the worst case for NMOS transistors.

HCI reliability strongly depends on the device architecture, especially on the effective gate length $\mathrm{L}_{\mathrm{eff}}$, the channel-drain junction, the spacer type, and the oxide thickness [16]. The proposed process optimisation reduces the gate oxide thickness, thus increases the gate oxide electric field transversal to the channel. This should be a disadvantage for our new MV transistor compared to the HV transistor, which is subjected to a more important electric field for the same polarisation.

HCI degradation evaluation has been performed on transistors having the minimal length $\mathrm{L}_{\min }$, in our case $0.7 \mu \mathrm{m}$, with a $\mathrm{W}$ of $10 \mu \mathrm{m}$. Initial characterizations of the transistors are made before any HCI stress, by extracting their initial $\mathrm{V}_{\mathrm{T}}$, and $\mathrm{I}_{\mathrm{ON}}$. The transistors are then stressed based on a half-decade time step interval pattern, the degradation being known to follow a powerlaw with time. The cumulative stress times is 1,310 , $30 \mathrm{~s}$, etc. and between each stress interval a characterization is made to track the devices parameters degradation over time [17]. Stress duration went up to $100000 \mathrm{~s}$.

For device qualification, the device lifetime is usually defined as the stress time necessary to cause a $10 \%$ drift of the ION current or a $100 \mathrm{mV}$ drift of the threshold voltage.

\subsection{Experimental results}

The new MV transistor will be subjected to an absolute maximum $\mathrm{V}_{\mathrm{DS}}$ voltage of $4.8 \mathrm{~V}$ for the NMOS and $-4.8 \mathrm{~V}$ for the PMOS. For this reason, we will perform the HCI stress at this $\mathrm{V}_{\mathrm{DS}}$ voltage. Corresponding to this $\mathrm{V}_{\mathrm{DS}}$ voltage, the $\mathrm{V}_{\mathrm{GS}}$ voltage of worst degradation must be selected. Fig. 5 a shows the $\mathrm{I}_{\mathrm{ON}}$ current degradation after preliminary HCI stresses of $1000 \mathrm{~s}$ for different $\mathrm{V}_{\mathrm{GS}}$ voltages for the MV and $\mathrm{HV}$ NMOS transistors. This measurement shows that the strongest degradation for $\mathrm{V}_{\mathrm{DS}}=4.8 \mathrm{~V}$ for both NMOS transistors occurs at $\mathrm{V}_{\mathrm{GS}}=2 \mathrm{~V}$ for the $\mathrm{MV}$ transistor and $3 \mathrm{~V}$ for the HV transistor. For the PMOS transistors, the $\mathrm{HCI}$ stress will be performed at maximum gate voltage, in our case $\mathrm{V}_{\mathrm{GS}}=\mathrm{V}_{\mathrm{DS}}=-4.8 \mathrm{~V}$, which has been verified 
to inflict the maximum damage on PMOS transistors.

Fig. $5 b, c, d, e$ shows the evolution of the device parameter $\left(\mathrm{I}_{\mathrm{ON}}\right.$ and $\left.\mathrm{V}_{\mathrm{T}}\right)$ versus the cumulative stress time. The evolution for PMOS transistors is shown in absolute value. A decrease of $\mathrm{I}_{\mathrm{ON}}$ drive current over stress time is observed for all the transistors, and an increase of $\mathrm{V}_{\mathrm{T}}$ during stress time is observed. Power-law fits are added to the HCI measurement data using the least-squares method (dashed lines on Fig. 5b,c,d,e). After $50000 \mathrm{~s}$ of $\mathrm{HCI}$ stress, the $\mathrm{V}_{\mathrm{T}}$ degradation is equal to $20 \mathrm{mV}$ for the HV NMOS, $30 \mathrm{mV}$ for the MV NMOS, $10 \mathrm{mV}$ for the HV PMOS and $13 \mathrm{mV}$ for the MV PMOS. The $\mathrm{I}_{\mathrm{ON}}$ degradation after $50000 \mathrm{~s}$ of HCI stress is equal to $-2 \%$ for the HV NMOS, $-4.25 \%$ for the MV NMOS, $-1.5 \%$ for the HV PMOS and $-4 \%$ for the MV PMOS. The oxide reduction associated with the new MV transistor has moderately decreased its lifetime.

The HCI reliability results of our new MV transistor are comparable with those of the HV transistor (with a slight advantage for the HV transistor), posing no reliability issues at the studied voltage and gate length.

\section{Perspectives}

Although the new MV transistor has very interesting performances at zero-cost, there are still some electrical parameters that can be improved upon regarding SCE. Firstly, because of SCE, the $\mathrm{V}_{\mathrm{T}}$ undergoes a roll-off that limits the transistor length to a $\mathrm{L}_{\min }$ value around $0.7 \mu \mathrm{m}$. This is accompanied by a leakage current increase that can cause problems in low-power applications. To try to solve these issues, TCAD and silicon investigations are ongoing to optimize the LDD regions of our new MV transistor, which are inherited from the HV transistor and not necessarily optimized for the MV range. Future work will consist in investigating several other LDD recipes from other devices from the technology that may be reused at zero-cost in our MV device, such as the LV transistors, the SRAM and flash memory devices. We will be particularly interested in the reliability of these optimisations.

\section{Conclusion}

A process optimisation is proposed to build a new "zero-cost" device able to address the MV range $(3-5 \mathrm{~V})$ in an eNVM technology for low-cost products. The proposed device is intended to replace a regular HV device that is currently used instead of a dedicated MV transistor to reduce cost by minimizing the number of photomasks and fabrication process steps. The process optimization resulting in this new MV transistor lowers the $\mathrm{V}_{\mathrm{T}}$ and increases the $\mathrm{I}_{\mathrm{ON}}$ at the cost of an increased $\mathrm{I}_{\mathrm{OFF}}$ compared to the HV device. It also enhances the matching performances, which is highly desirable for analogue applications. Hot-carrier degradation evaluation shows a slightly shorter lifetime compared to the HV device, but no reliability issues.

\section{References}

[1] W. Sun et al., "Low-cost embedded Flash memory technology", 2011 IEEE International Conference on IC Design \& Technology, Kaohsiung, pp. 1-5, 2011.

[2] P. Cappelletti et al., "Flash memories", Springer Science \& Business Media, 2013.

[3] M. Pelgrom, A. Duinmaijer and A. Welbers "Matching properties of MOS transistors", IEEE Journal of SolidState Circuits vol. SC-24 pp. 1433-1440, 1989.

[4] Y. Joly et al., "Impact of hump effect on MOSFET mismatch in the sub-threshold area for low power analog applications", International Conference on Solid-State and Integrated Circuit Technology, 2010.

[5] Synopsys ${ }^{\circledR}$, "Sentaurus Process", Version O-2018-06

[6] Synopsys ${ }^{\circledR}$, “Sentaurus Device”, Version O-2018-06

[7] D. Klaassen, "A Unified Mobility Model for Device Simulation-I. Model Equations and Concentration Dependence", in Solid-State Electronics, vol. 35, no. 7, pp. 953-959, 1992.

[8] E. Gnani, S. Reggiani, M. Rudan and G. Baccarani, "Extraction Method for the Impact-Ionization Multiplication Factor in Silicon at Large Operating Temperatures", 32nd European Solid-State Device Research Conference, pp. 227-230, 2002

[9] Y. Taur et al., "CMOS scaling into the nanometer regime", in Proceedings of the IEEE, vol. 85, no. 4, pp. 486-504, 1997

[10] K. Roy et al., "Leakage current mechanisms and leakage reduction techniques in deep-submicrometer CMOS circuits", in Proceedings of the IEEE, vol. 91, no. 2, 2003

[11] Y. Yamaguchi et al., "Source-to-drain breakdown voltage improvement in ultrathin-film SOI MOSFET's using a gate-overlapped LDD structure", in IEEE Transactions on Electron Devices, vol. 41, no. 7, pp. 1222-1226, 1994

[12] W. Jiang "Hot-carrier reliability assessment in CMOS digital integrated circuits" Thesis - Massachusetts Institute of Technology, Dept. of Electrical Engineering and Computer Science, 1998.

[13] M. Thomason, J. Prasad and J. De Greve, "Suppression of the reverse short channel effect in sub-micron CMOS devices", International Semiconductor Device Research Symposium, 2003, Washington, DC, USA, 2003, pp. 420421.

[14] E. Takeda, C. Yang, A. Miura-Hamada, "Hot-carrier effects in MOS devices", Academic Press, 1995.

[15] J. Bernstein "Failure Mechanisms", Academic Press, 2014

[16] Y. Joly et al., "Matching degradation of threshold voltage and gate voltage of NMOSFET after Hot Carrier Injection stress." microelectronics reliability 51.9-11, 1561-1563, 2011

[17] JEDEC standard 28-A "Procedure for Measuring NChannel MOSFET Hot-Carrier-Induced Degradation Under DC Stress", 2001 